TRANSACTIONS OF THE

AMERICAN MATHEMATICAL SOCIETY

Volume 355, Number 3, Pages 925-933

S 0002-9947(02)03132-X

Article electronically published on November 5, 2002

\title{
SOME PROPERTIES OF THE SCHOUTEN TENSOR AND APPLICATIONS TO CONFORMAL GEOMETRY
}

\author{
PENGFEI GUAN, JEFF VIACLOVSKY, AND GUOFANG WANG
}

\begin{abstract}
The Riemannian curvature tensor decomposes into a conformally invariant part, the Weyl tensor, and a non-conformally invariant part, the Schouten tensor. A study of the $k$ th elementary symmetric function of the eigenvalues of the Schouten tensor was initiated in an earlier paper by the second author, and a natural condition to impose is that the eigenvalues of the Schouten tensor are in a certain cone, $\Gamma_{k}^{+}$. We prove that this eigenvalue condition for $k \geq n / 2$ implies that the Ricci curvature is positive. We then consider some applications to the locally conformally flat case, in particular, to extremal metrics of $\sigma_{k}$-curvature functionals and conformal quermassintegral inequalities, using the results of the first and third authors.
\end{abstract}

\section{INTRODUCTION}

Let $\left(M^{n}, g\right)$ be an $n$-dimensional Riemannian manifold, $n \geq 3$, and let the Ricci tensor and scalar curvature be denoted by Ric and $R$, respectively. We define the Schouten tensor

$$
A_{g}=\frac{1}{n-2}\left(R i c-\frac{1}{2(n-1)} R g\right) .
$$

There is a decomposition formula (see [1]):

$$
\text { Riem }=A_{g} \odot g+\mathcal{W}_{g}
$$

where $\mathcal{W}_{g}$ is the Weyl tensor of $g$, and $\odot$ denotes the Kulkarni-Nomizu product (see [1]). Since the Weyl tensor is conformally invariant, to study the deformation of the conformal metric, we only need to understand the Schouten tensor. A study of $k$-th elementary symmetric functions of the Schouten tensor was initiated in [13], it was reduced to certain fully nonlinear Yamabe type equations. In order to apply the elliptic theory of fully nonlinear equations, one often restricts the Schouten tensor to be in a certain cone $\Gamma_{k}^{+}$, defined as follows (according to Gärding [5]).

Definition 1. Let $\left(\lambda_{1}, \cdots, \lambda_{n}\right) \in \mathbf{R}^{n}$. Let $\sigma_{k}$ denote the $k$ th elementary symmetric function

$$
\sigma_{k}\left(\lambda_{1}, \cdots, \lambda_{n}\right)=\sum_{i_{1}<\cdots<i_{k}} \lambda_{i_{1}} \cdots \lambda_{i_{k}}
$$

Received by the editors April 19, 2002.

2000 Mathematics Subject Classification. Primary 53C21; Secondary 35J60, 58E11.

Key words and phrases. $\Gamma_{k}$-curvature, Ricci curvature, conformal deformation.

Research of the first author was supported in part by NSERC Grant OGP-0046732.

Research of the second author was supported in part by an NSF Postdoctoral Fellowship.

(C)2002 American Mathematical Society 
and let

$$
\Gamma_{k}^{+}=\text {component of }\left\{\sigma_{k}>0\right\} \text { containing }(1, \cdots, 1) .
$$

Let $\bar{\Gamma}_{k}^{+}$denote the closure of $\Gamma_{k}^{+}$. If $(M, g)$ is a Riemannian manifold, and $x \in M$, we say $g$ has positive (nonnegative, resp.) $\Gamma_{k}$-curvature at $x$ if its Schouten tensor $A_{g} \in \Gamma_{k}^{+}\left(\bar{\Gamma}_{k}^{+}\right.$, resp. $)$at $x$. In this case, we also say $g \in \Gamma_{k}^{+}\left(\bar{\Gamma}_{k}^{+}\right.$, resp. $)$at $x$.

We note that positive $\Gamma_{1}$-curvature is equivalent to positive scalar curvature, and the condition of positive $\Gamma_{k}$-curvature has some geometric and topological consequences for the manifold $M$. For example, when $(M, g)$ is locally conformally flat with positive $\Gamma_{1}$-curvature, then $\pi_{i}(M)=0, \forall 1<i \leq \frac{n}{2}$, by a result of Schoen and Yau [11]. In this note, we will prove that positive $\Gamma_{k}$-curvature for any $k \geq \frac{n}{2}$ implies positive Ricci curvature.

Theorem 1. Let $(M, g)$ be a Riemannian manifold and $x \in M$. If $g$ has positive (nonnegative, resp.) $\Gamma_{k}$-curvature at $x$ for some $k \geq n / 2$, then its Ricci curvature is positive (nonnegative, resp.) at $x$. Moreover, if the $\Gamma_{k}$-curvature is nonnegative for some $k>1$, then

$$
\operatorname{Ric}_{g} \geq \frac{2 k-n}{2 n(k-1)} R_{g} \cdot g .
$$

In particular, if $k \geq \frac{n}{2}$, then

$$
R i c_{g} \geq \frac{(2 k-n)(n-1)}{(k-1)}\left(\begin{array}{l}
n \\
k
\end{array}\right)^{-\frac{1}{k}} \sigma_{k}^{\frac{1}{k}}\left(A_{g}\right) \cdot g .
$$

Remark. Theorem 1 is not true for $k=1$. Namely, the condition of positive scalar curvature gives no restriction on the lower bound of the Ricci curvature.

Corollary 1. Let $\left(M^{n}, g\right)$ be a compact, locally conformally flat manifold with nonnegative $\Gamma_{k}$-curvature everywhere for some $k \geq n / 2$. Then $(M, g)$ is conformally equivalent to either a space form or a finite quotient of a Riemannian $\mathbf{S}^{n-1}(c) \times \mathbf{S}^{1}$ for some constant $c>0$ and $k=n / 2$. In particular, if $g \in \Gamma_{k}^{+}$, then $(M, g)$ is conformally equivalent to a spherical space form.

When $n=3,4$, the result in Theorem [1 was already observed in [9] and [2]. Theorem 1 and Corollary 1 will be proved in the next section.

We will also consider the equation

$$
\sigma_{k}\left(A_{\tilde{g}}\right)=\text { constant }
$$

for conformal metrics $\tilde{g}=e^{-2 u} g$. This equation was studied in [13, where it was shown that when $k \neq n / 2$, (2) is the conformal Euler-Lagrange equation of the functional

$$
\mathcal{F}_{k}(g)=\operatorname{Vol}(g)^{-\frac{n-2 k}{n}} \int_{M} \sigma_{k}(g) d \operatorname{vol}(g),
$$

when $k=1,2$ or for $k>2$ when $M$ is locally conformally flat. We remark that in the even-dimensional locally conformally flat case, $\mathcal{F}_{n / 2}$ is a conformal invariant. Moreover, it is a multiple of the Euler characteristic, see [13].

This problem was further studied in [7, where the following conformal flow was considered:

$$
\begin{aligned}
\frac{d}{d t} g & =-\left(\log \sigma_{k}(g)-\log r_{k}(g)\right) \cdot g, \\
g(0) & =g_{0},
\end{aligned}
$$


where

$$
\log r_{k}=\frac{1}{\operatorname{Vol}(g)} \int_{M} \log \sigma_{k}(g) d \operatorname{vol}(g) .
$$

Global existence with uniform $C^{1,1}$ a priori bounds of the flow was proved in [7]. It was also proved that for $k \neq n / 2$ the flow is sequentially convergent in $C^{1, \alpha}$ to a $C^{\infty}$ solution of $\sigma_{k}=$ constant. Also, if $k<n / 2$, then $\mathcal{F}_{k}$ is decreasing along the flow, and if $k>n / 2$, then $\mathcal{F}_{k}$ is increasing along the flow. We remark that the existence result for equation (2) has been obtained independently in 10 in the locally conformally flat case for all $k$.

In Section 3, we will consider global properties of the functional $\mathcal{F}_{k}$, and give conditions for the existence of a global extremizer. We will also derive some conformal quermassintegral inequalities, which are analogous to the classical quermassintegral inequalities in convex geometry.

\section{Curvature Restriction}

We first state a proposition which describes some important properties of the $\operatorname{sets} \Gamma_{k}^{+}$.

Proposition 1. (i) Each set $\Gamma_{k}^{+}$is an open convex cone with vertex at the origin, and we have the following sequence of inclusions:

$$
\Gamma_{n}^{+} \subset \Gamma_{n-1}^{+} \subset \cdots \subset \Gamma_{1}^{+} \text {. }
$$

(ii) For any $\Lambda=\left(\lambda_{1}, \cdots, \lambda_{n}\right) \in \Gamma_{k}^{+}\left(\bar{\Gamma}_{k}^{+}\right.$, resp. $), \forall 1 \leq i \leq n$, let

$$
(\Lambda \mid i)=\left(\lambda_{1}, \cdots, \lambda_{i-1}, \lambda_{i+1}, \cdots, \lambda_{n}\right) .
$$

Then $(\Lambda \mid i) \in \Gamma_{k-1}^{+}\left(\bar{\Gamma}_{k-1}^{+}\right.$, resp. $)$. In particular,

$$
\Gamma_{n-1}^{+} \subset V_{n-1}^{+}=\left\{\left(\lambda_{1}, \cdots, \lambda_{n}\right) \in \mathbf{R}^{n}: \lambda_{i}+\lambda_{j}>0, i \neq j\right\} .
$$

The proof of this proposition is standard, following from [5].

Our main results are consequences of the following two lemmas. In this note, we assume that $k>1$.

Lemma 1. Let $\Lambda=\left(\lambda_{1}, \lambda_{2}, \cdots, \lambda_{n-1}, \lambda_{n}\right) \in \mathbb{R}^{n}$, and define

$$
A_{\Lambda}=\Lambda-\frac{\sum_{i=1}^{n} \lambda_{i}}{2(n-1)}(1,1, \cdots, 1) \text {. }
$$

If $A_{\Lambda} \in \bar{\Gamma}_{k}^{+}$, then

$$
\min _{i=1, \cdots, n} \lambda_{i} \geq \frac{(2 k-n)}{2 n(k-1)} \sum_{i=1}^{n} \lambda_{i} .
$$

In particular, if $k \geq \frac{n}{2}$, then

$$
\min _{i=1, \cdots, n} \lambda_{i} \geq \frac{(2 k-n)(n-1)}{(n-2)(k-1)}\left(\begin{array}{l}
n \\
k
\end{array}\right)^{-\frac{1}{k}} \sigma_{k}^{\frac{1}{k}}\left(A_{\Lambda}\right) .
$$

Proof. We first note that, for any nonzero vector $A=\left(a_{1}, \cdots, a_{n}\right) \in \bar{\Gamma}_{2}^{+}$we have $\sigma_{1}(A)>0$. This can be proved as follows. Since $A \in \bar{\Gamma}_{2}^{+}, \sigma_{1}(A) \geq 0$. If $\sigma_{1}(A)=0$, there must be an $a_{i}>0$ for some $i$, since $A$ is a nonzero vector. We may assume $a_{n}>0$. Let $(A \mid n)=\left(a_{1}, \cdots, a_{n-1}\right)$; we have $\sigma_{1}(A \mid n) \geq 0$ by Proposition 11. This would give $\sigma_{1}(A)=\sigma_{1}(A \mid n)+a_{n}>0$, a contradiction. 
Now without loss of generality, we may assume that $\Lambda$ is not a zero vector. By the assumption $A_{\Lambda} \in \bar{\Gamma}_{k}^{+}$for $k \geq 2$, so we have $\sum_{i=1}^{n} \lambda_{i}>0$.

Define

$$
\Lambda_{0}=\left(1,1, \cdots, 1, \delta_{k}\right) \in \mathbb{R}^{n-1} \times \mathbb{R}
$$

then we have $A_{\Lambda_{0}}=(a, \cdots, a, b)$, where

so that

$$
\begin{gathered}
\delta_{k}=\frac{(2 k-n)(n-1)}{2 n k-2 k-n}, \\
a=1-\frac{n-1+\delta_{k}}{2(n-1)}, \quad b=\delta_{k}-\frac{n-1+\delta_{k}}{2(n-1)},
\end{gathered}
$$

$$
\sigma_{k}\left(A_{\Lambda_{0}}\right)=0 \quad \text { and } \sigma_{j}\left(A_{\Lambda_{0}}\right)>0 \text { for } j \leq k-1 .
$$

It is clear that $\delta_{k}<1$, and so $a>b$. Since (44) is invariant under the transformation from $\Lambda$ to $s \Lambda$ for $s>0$, we may assume that $\sum_{i=1}^{n} \lambda_{i}=\operatorname{tr}\left(\Lambda_{0}\right)=n-1+\delta_{k}$ and $\lambda_{n}=\min _{i=1, \cdots, n} \lambda_{i}$. We write

$$
A_{\Lambda}=\left(a_{1}, \cdots, a_{n}\right)
$$

We claim that

$$
\lambda_{n} \geq \delta_{k} .
$$

This is equivalent to showing

$$
a_{n} \geq b \text {. }
$$

Assume for a contradiction that $a_{n}<b$. We consider $\Lambda_{t}=t \Lambda_{0}+(1-t) \Lambda$ and

$$
\begin{aligned}
A_{t}:=A_{\Lambda_{t}} & =t A_{\Lambda_{0}}+(1-t) A_{\Lambda} \\
& =\left((1-t) a+t a_{1}, \cdots,(1-t) a+t a_{n-1},(1-t) b+t a_{n}\right) .
\end{aligned}
$$

By the convexity of the cone $\Gamma_{k}^{+}$(see Proposition 1), we know that

$$
A_{t} \in \bar{\Gamma}_{k}^{+}, \quad \text { for any } t \in(0,1] \text {. }
$$

In particular, $f(t):=\sigma_{k}\left(A_{t}\right) \geq 0$ for any $t \in[0,1]$. By the definition of $\delta_{k}, f(0)=0$.

For any $i$ and any vector $V=\left(v_{1}, \cdots, v_{n}\right)$, we denote by

$$
(V \mid i)=\left(v_{1}, \cdots, v_{i-1}, v_{i+1}, \cdots, v_{n}\right)
$$

the vector with the $i$-th component removed. Now we compute the derivative of $f$ at 0:

$$
f^{\prime}(0)=\sum_{i=1}^{n-1}\left(a_{i}-a\right) \sigma_{k-1}\left(A_{0} \mid i\right)+\left(a_{n}-b\right) \sigma_{k-1}\left(A_{0} \mid n\right) .
$$

Since $\left(A_{0} \mid i\right)=\left(A_{0} \mid 1\right)$ for $i \leq n-1$ and $\sum_{i=1}^{n} a_{i}=(n-1) a+b$, we have

$$
f^{\prime}(0)=\left(a_{n}-b\right)\left(\sigma_{k-1}\left(A_{0} \mid n\right)-\sigma_{k-1}\left(A_{0} \mid 1\right)\right)<0,
$$

for $\sigma_{k-1}\left(A_{0} \mid n\right)-\sigma_{k-1}\left(A_{0} \mid 1\right)>0$. (Recall that $b<a$.) This is a contradiction; hence $\lambda_{n} \geq \delta_{k}$. It follows that

$$
\min _{i=1, \cdots, n} \lambda_{i} \geq \delta_{k}=\frac{2 k-n}{2 n(k-1)} \sum_{i=1}^{n} \lambda_{i} .
$$

Finally, the last inequality in the lemma follows from the Newton-MacLaurin inequality. 
Remark. It is clear from the above proof that the constant in Lemma 1 is optimal.

We next consider the case $A_{\Lambda} \in \bar{\Gamma}_{\frac{n}{2}}^{+}$.

Lemma 2. Let $k=n / 2$ and $\Lambda=\left(\lambda_{1}, \cdots, \lambda_{n}\right) \in \mathbb{R}^{n}$ with $A_{\Lambda} \in \bar{\Gamma}_{k}^{+}$. Then either $\lambda_{i}>0$ for any $i$, or

$$
\Lambda=(\lambda, \lambda, \cdots, \lambda, 0)
$$

up to a permutation. If the second case is true, then we must have $\sigma_{\frac{n}{2}}\left(A_{\Lambda}\right)=0$.

Proof. By Lemma 1 to prove the Lemma we only need to check that for $\Lambda=$ $\left(\lambda_{1}, \cdots, \lambda_{n-1}, 0\right)$ with $A_{\Lambda} \in \bar{\Gamma}_{k}^{+}$,

$$
\lambda_{i}=\lambda_{j}, \quad \forall i, j=1,2, \cdots, 2 k-1 .
$$

We use the same idea as in the proof of the previous Lemma. Without loss of generality, we may assume that $\Lambda$ is not a zero vector. By the assumption $A_{\Lambda} \in \bar{\Gamma}_{k}^{+}$ for $k \geq 2$, we have $\sum_{i=1}^{n-1} \lambda_{i}>0$. Hence we may assume that $\sum_{i=1}^{n-1} \lambda_{i}=n-1$. Define

$$
\Lambda_{0}=(1,1, \cdots, 1,0) \in \mathbb{R}^{n}
$$

It is easy to check that

$$
A_{\Lambda_{0}} \in \Gamma_{k-1}^{+} \quad \text { and } \quad \sigma_{k}\left(A_{\Lambda_{0}}\right)=0 .
$$

That is, $A_{\Lambda_{0}} \in \bar{\Gamma}_{k}^{+}$. If the $\lambda$ 's are not all the same, we have

$$
\sum_{i=1}^{n-1}\left(\lambda_{i}-1\right)=0
$$

and

$$
\sum_{i=1}^{n-1}\left(\lambda_{i}-1\right)^{2}>0
$$

Now consider $\Lambda_{t}=t \Lambda_{0}+(1-t) \Lambda$ and

$$
A_{t}:=A_{\Lambda_{t}}=t A_{\Lambda_{0}}+(1-t) A_{\Lambda}=\left(\frac{1}{2}+t\left(\lambda_{1}-1\right), \cdots, \frac{1}{2}+t\left(\lambda_{n-1}-1\right),-\frac{1}{2}\right) .
$$

From the assumption that $A \in \bar{\Gamma}_{k}^{+},(8)$, and the convexity of $\bar{\Gamma}_{k}^{+}$, we have

$$
A_{t} \in \bar{\Gamma}_{k}^{+} \quad \text { for } t>0 \text {. }
$$

For any $i \neq j$ and any vector $A$, we denote by $(A \mid i j)$ the vector with the $i$-th and $j$-th components removed. Let $\widetilde{\Lambda}=\left(\frac{1}{2}, \cdots, \frac{1}{2},-\frac{1}{2}\right)$ be an $(n-1)$-vector, and $\Lambda^{*}=\left(\frac{1}{2}, \cdots, \frac{1}{2},-\frac{1}{2}\right)$ an $(n-2)$-vector. It is clear that $\forall i \neq j, \quad i, j \leq n-1$,

$$
\begin{gathered}
\sigma_{k-1}\left(A_{0} \mid i\right)=\sigma_{k-1}(\widetilde{\Lambda})>0, \\
\sigma_{k-2}\left(A_{0} \mid i j\right)=\sigma_{k-2}\left(\Lambda^{*}\right)>0 .
\end{gathered}
$$

Now we expand $f(t)=\sigma_{k}\left(A_{t}\right)$ at $t=0$. By (8), $f(0)=0$. We compute

$$
\begin{aligned}
f^{\prime}(0) & =\sum_{i=1}^{n-1}\left(\lambda_{i}-1\right) \sigma_{k-1}\left(A_{0} \mid i\right) \\
& =\sigma_{k-1}(\widetilde{\Lambda}) \sum_{i=1}^{n-1}\left(\lambda_{i}-1\right)=0
\end{aligned}
$$


and

$$
\begin{aligned}
f^{\prime \prime}(0) & =\sum_{i \neq j}\left(\lambda_{i}-1\right)\left(\lambda_{j}-1\right) \sigma_{k-2}\left(A_{0} \mid i j\right) \\
& =\sigma_{k-2}\left(\Lambda^{*}\right) \sum_{i \neq j}\left(\lambda_{i}-1\right)\left(\lambda_{j}-1\right) \\
& =-\sigma_{k-2}\left(\Lambda^{*}\right) \sum_{i=1}^{n-1}\left(\lambda_{i}-1\right)^{2}<0,
\end{aligned}
$$

for $\sigma_{k-2}\left(A_{0} \mid i j\right)=\sigma_{k-2}\left(\Lambda^{*}\right)>0$ for any $i \neq j$ and $\sum_{i \neq j}\left(\lambda_{j}-1\right)=\left(1-\lambda_{i}\right)$. Hence $f(t)<0$ for small $t>0$, which contradicts (9).

Remark. From the proof of Lemma 2 , there is a constant $C>0$, depending only on $n$ and $\frac{\sigma_{\frac{n}{2}}^{\frac{2}{n}}\left(A_{\Lambda}\right)}{\sigma_{1}\left(A_{\Lambda}\right)}$, such that

$$
\min _{i} \lambda_{i} \geq C \sigma_{\frac{n}{2}}^{\frac{2}{n}}\left(A_{\Lambda}\right)
$$

Proof of Theorem 1. Theorem 1 follows directly from Lemmas 1 and 2

Corollary 2. Let $(M, g)$ be an $n$-dimensional Riemannian manifold and $k \geq n / 2$, and let $N=M \times \mathbf{S}^{1}$ be the product manifold. Then $N$ does not have positive $\Gamma_{k}$ curvature. If $N$ has nonnegative $\Gamma_{k}$-curvature, then $(M, g)$ is an Einstein manifold, and there are two cases: either $k=n / 2$, or $k>n / 2$ and $(M, g)$ is a torus.

Proof. This follows from Lemmas 1 and 2 .

Proof of Corollary 1 From Theorem 1, we know that the Ricci curvature Ric $c_{g}$ is nonnegative. Now we deform it by the Yamabe flow considered by Hamilton, Ye [15] and Chow 44 to obtain a conformal metric $\widetilde{g}$ of constant scalar curvature. The Ricci curvature $\operatorname{Ric}_{\tilde{g}}$ is nonnegative, for the Yamabe flow preserves the nonnegativity of the Ricci curvature, see [4]. Now, by a classification result given in [12, 3], we know that $(M, \widetilde{g})$ is isometric to either a space form or a finite quotient of a Riemannian $\mathbf{S}^{n-1}(c) \times \mathbf{S}^{1}$ for some constant $c>0$. In the latter case, it is clear that $k=n / 2$, since otherwise it cannot have nonnegative $\Gamma_{k}$-curvature.

Next, we will prove that if $M$ is locally conformally flat with positive $\Gamma_{n-1^{-}}$ curvature, then $g$ has positive sectional curvature. If $M$ is locally conformally flat, then by (1) we may decompose the full curvature tensor as

$$
\text { Riem }=A_{g} \odot g,
$$

Proposition 2. Assume that $n=3$, or that $M$ is locally conformally flat. Then the Schouten tensor $A_{g} \in V_{n-1}^{+}$if and only if $g$ has positive sectional curvature.

Proof. Let $\pi$ be any 2-plane in $T_{p}(N)$, and let $X, Y$ be an orthonormal basis of $\pi$. We have

$$
\begin{aligned}
K(\sigma)= & \operatorname{Riem}(X, Y, X, Y)=A_{g} \odot g(X, Y, X, Y) \\
= & A_{g}(X, X) g(Y, Y)-A_{g}(Y, X) g(X, Y) \\
& +A_{g}(Y, Y) g(X, X)-A_{g}(X, Y) g(Y, X) \\
= & A_{g}(X, X)+A_{g}(Y, Y) .
\end{aligned}
$$


From this it follows that

$$
\min _{\sigma \in T_{p} N} K(\sigma)=\lambda_{1}+\lambda_{2},
$$

where $\lambda_{1}$ and $\lambda_{2}$ are the smallest eigenvalues of $A_{g}$ at $p$.

Corollary 3. If $(M, g)$ is locally conformally flat with positive $\Gamma_{n-1}$-curvature, then $g$ has positive sectional curvature.

Proof. This follows easily from Propositions 1 and 2

\section{Extremal Metrics of $\sigma_{k}$-CURVATURE FUnCtionals}

We next consider some properties of the functionals $\mathcal{F}_{k}$ associated to $\sigma_{k}$. These functionals were introduced and discussed in [13], see also [7]. Further variational properties in connection to 3 -dimensional geometry were studied in 9 .

We recall that $\mathcal{F}_{k}$ is defined by

$$
\mathcal{F}_{k}(g)=\operatorname{Vol}(g)^{-\frac{n-2 k}{n}} \int_{M} \sigma_{k}(g) d \operatorname{vol}(g)
$$

We denote $\mathcal{C}_{k}=\left\{g \in\left[g_{0}\right] \mid g \in \Gamma_{k}^{+}\right\}$, where $\left[g_{0}\right]$ is the conformal class of $g_{0}$.

We now apply our results to show that if $g_{0} \in \Gamma_{\frac{n}{2}}^{+}$, then there is an extremal metric $g_{e}$ which minimizes $\mathcal{F}_{m}$ for $m<n / 2$, and if $m>n / 2$, there is an extremal metric $g_{e}$ which maximizes $\mathcal{F}_{m}$.

Proposition 3. Suppose $\left(M, g_{0}\right)$ is locally conformally flat and $g_{0} \in \Gamma_{k}^{+}$for some $k \geq \frac{n}{2}$. Then $\forall m<\frac{n}{2}$, there is an extremal metric $g_{e}^{m} \in\left[g_{0}\right]$ such that

$$
\inf _{g \in \mathcal{C}_{m}} \mathcal{F}_{m}(g)=\mathcal{F}_{m}\left(g_{e}^{m}\right)
$$

and $\forall m>\frac{n}{2}$, there is extremal metric $g_{e}^{m} \in\left[g_{0}\right]$ such that

$$
\sup _{g \in \mathcal{C}_{m}} \mathcal{F}_{k}(g)=\mathcal{F}_{k}\left(g_{e}^{m}\right) \text {. }
$$

In fact, any solution to $\sigma_{m}(g)=$ constant is an extremal metric.

Proof. First, by Corollary 1. $\left(M, g_{0}\right)$ is conformal to a spherical space form. For any $g \in \mathcal{C}_{m}$, from [7] we know there is a conformal metric $\widetilde{g}$ in $\mathcal{C}_{m}$ such that $\sigma_{m}(\widetilde{g})$ is constant and

(a) if $m>n / 2$, then $\mathcal{F}_{m}(g) \leq \mathcal{F}_{m}(\widetilde{g})$,

(b) if $m<n / 2$, then $\mathcal{F}_{m}(g) \geq \mathcal{F}_{m}(\widetilde{g})$.

A classification result of [13] and [14], which is analogous to a result of Obata for the scalar curvature, shows that $\widetilde{g}$ has constant sectional curvature. Therefore $\widetilde{g}$ is the unique critical metric unless $M$ is conformally equivalent to $\mathbf{S}^{n}$, in which case any critical metric is the image of the standard metric under a conformal diffeomorphism. This clearly implies the conclusion of the Proposition.

Next we consider the case $k<n / 2$. We have

Proposition 4. Suppose $\left(M, g_{0}\right)$ is locally conformally flat and $g_{0} \in \Gamma_{k}^{+}$for some $k<\frac{n}{2}$. Suppose furthermore that for any fixed $C>0$, the space of solutions to the equation $\sigma_{k}=C$ is compact, with a bound independent of the constant $C$. Then there is an extremal metric $g_{e}^{k} \in\left[g_{0}\right]$ such that

$$
\inf _{g \in \mathcal{C}_{k}} \mathcal{F}_{k}(g)=\mathcal{F}_{k}\left(g_{e}^{k}\right)
$$


Proof. From the compactness assumption, there exists a critical metric $g_{e}^{k}$ which has least energy. If the functional assumed a value strictly lower than $\mathcal{F}_{k}\left(g_{e}^{k}\right)$, then by [7], the flow would decrease to another solution of $\sigma_{k}=$ constant, which is a contradiction since $g_{e}^{k}$ has minimal energy.

We conclude with conformal quermassintegral inequalities, which were conjectured in [7, and verified there for some special cases when $(M, g)$ is locally conformally flat and $g \in \Gamma_{\frac{n}{2}-1}^{+}$or $g \in \Gamma_{\frac{n}{2}+1}^{+}$using the flow method. In the case of $k=2, n=4$, the inequality was proved in 8 , without the locally conformally flat assumption.

Proposition 5. Suppose $\left(M, g_{0}\right)$ is locally conformally flat and $g_{0} \in \Gamma_{k}^{+}$for some $k \geq \frac{n}{2}$. Then for any $1 \leq l<\frac{n}{2} \leq k \leq n$ there is a constant $C(k, l, n)>0$ such that for any $g \in\left[g_{0}\right]$ and $g \in \Gamma_{k}^{+}$,

$$
\left(\mathcal{F}_{k}(g)\right)^{1 / k} \leq C(k, l, n)\left(\mathcal{F}_{l}(g)\right)^{1 / l},
$$

with equality if and only if $(M, g)$ is a spherical space form.

Proof. By Proposition 3, we have a conformal metric $g_{e}$ of constant sectional curvature such that

$$
\inf _{g \in \mathcal{C}_{l}} \mathcal{F}_{l}(g)=\mathcal{F}_{l}\left(g_{e}\right)
$$

and

$$
\sup _{g \in \mathcal{C}_{k}} \mathcal{F}_{k}(g)=\mathcal{F}_{k}\left(g_{e}\right)
$$

Hence, for any $g \in \Gamma_{k}^{+}$we have

$$
\begin{aligned}
\frac{\left(\mathcal{F}_{k}(g)\right)^{1 / k}}{\left(\mathcal{F}_{l}(g)\right)^{1 / l}} & \leq \frac{\left(\mathcal{F}_{k}\left(g_{e}\right)\right)^{1 / k}}{\left(\mathcal{F}_{l}\left(g_{e}\right)\right)^{1 / l}} \\
& =\frac{(l !(n-l) !)^{1 / l}}{(k !(n-k) !)^{1 / k}}
\end{aligned}
$$

When the equality holds, $g$ is an extremal of $\mathcal{F}_{l}$, hence a metric of constant sectional curvature by 13 .

\section{REFERENCES}

[1] A. Besse, Einstein manifolds, Springer-Verlag, Berlin, 1987. MR 88f:53087

[2] A. Chang, M. Gursky and P. Yang, An equation of Monge-Ampère type in conformal geometry, and four manifolds of positive Ricci curvature, to appear in Ann. of Math.

[3] Q.-M. Cheng, Compact locally conformally flat Riemannian manifolds. Bull. London Math. Soc. 33 (2001), no. 4, 459-465. MR 2002g:53045

[4] B. Chow, The Yamabe flow on locally conformally flat manifolds with positive Ricci curvature, Comm. Pure Appl. Math. 45 (1992), no. 8, 1003-1014. MR 93d:53045

[5] L. Gärding, An inequality for hyperbolic polynomials, J. Math. Mech. 8 (1959), 957-965. MR 22:4809

[6] P. Guan and G. Wang, Local estimates for a class of fully nonlinear equations arising from conformal geometry, preprint, August, 2001.

[7] P. Guan and G. Wang, A fully nonlinear conformal flow on locally conformally flat manifolds, preprint, October, 2001.

[8] M. Gursky, The principal eigenvalue of a conformally invariant differential operator, with an application to semilinear elliptic PDE, Comm. Math. Phys. 207 (1999), 131-143. MR 2000k:58029 
[9] Matthew J. Gursky and Jeff A. Viaclovsky, A new variational characterization of threedimensional space forms, Inventiones Mathematicae 145 (2001), no. 2, 251-278. MR 2002j:53039

[10] A. Li and Y. Li, On some conformally invariant fully nonlinear equations, C. R. Math. Acad. Sci. Paris 334 (2002), 305-310.

[11] R. Schoen and S. T. Yau, Conformally flat manifolds, Kleinian groups and scalar curvature, Invent. Math. 92 (1988), no. 1, 47-71. MR 89c:58139

[12] M. Tani, On a conformally flat Riemannian space with positive Ricci curvature. Tohoku Math. J. (2) 191967 227-231. MR 36:3279

[13] Jeff A. Viaclovsky, Conformal geometry, contact geometry and the calculus of variations, Duke J. Math. 101 (2000), no. 2, 283-316. MR 2001b:53038

[14] Jeff A. Viaclovsky, Conformally invariant Monge-Ampère equations: global solutions, Trans. Amer. Math. Soc. 352 (2000), no. 9, 4371-4379. MR 2000m:35067]

[15] R. Ye, Global existence and convergence of Yamabe flow, J. Differential Geom. 39 (1994), no. 1, 35-50. MR 95d:53044

Department of Mathematics, McMaster University, Hamilton, Ontario L8S 4K1, CANADA

E-mail address: guan@math.mcmaster.ca

Department of Mathematics, Massachusetts institute of Technology, Cambridge, Massachusetts

E-mail address: jeffv@math.mit.edu

Max-Planck-Institute for Mathematics in the Sciences, Inselstrasse 22-26, 04103 LEIPZIG, GeRmany

E-mail address: gwang@mis.mpg.de 